\title{
Seletividade de agrotóxicos utilizados na cultura do arroz irrigado ao fungo Metarhizium anisopliae, agente de controle microbiano de Tibraca limbativentris
}

\author{
Selectivity of chemical pesticides used in rice irrigated crop at fungus Metarhizium anisopliae, \\ microbial control agent of Tibraca limbativentris
}

\author{
Fátima Teresinha Rampelotti-Ferreira' ${ }^{\mathrm{I}}$ Anderson Ferreira ${ }^{\mathrm{II}}$ Honório Francisco Prando ${ }^{\mathrm{III}}$ \\ Fernando Adami Tcacenco ${ }^{\mathrm{III}}$ Anderson Dionei Grützmacher ${ }^{\mathrm{IV}}$ José Francisco da Silva Martins $^{\mathrm{V}}$
}

RESUMO

Para subsidiar a utilização de táticas de manejo integrado para o inseto-praga Tibraca limbativentris, avaliouse a compatibilidade in vitro entre o isolado CG 891 de Metarhizium anisopliae e os agrotóxicos utilizados na cultura do arroz irrigado. Os parâmetros avaliados foram crescimento vegetativo, esporulação e germinação em pré e pós-contato do fungo, com duas doses de fipronil, tiametoxam, fenitrotiona, carbofurano, pirazossulfurom-etílico, bentazona, clomazona, glifosato e quincloraque; e uma dose de triciclazol e azoxistrobina. Reduções significativas no crescimento vegetativo e esporulação de M. anisopliae foram observadas para fenitrotiona, carbofurano e azoxistrobina. Observou-se redução na germinação em pré-contato para o agrotóxico azoxistrobina. $O$ valor percentual da germinação alterou a classificação dos agrotóxicos com relação a sua seletividade sobre o entomopatógeno. No entanto, os resultados mostram que in vitro os agrotóxicos fenitrotiona, carbofurano, glifosato e azoxistrobina são prejudiciais ao isolado CG 891 de $\mathbf{M}$. anisopliae.

Palavras-chave: arroz irrigado, entomopatógeno, manejo integrado de pragas, percevejo-do-colmo, toxicidade.

\section{ABSTRACT}

Searching for integrated control strategies for the insect-pest Tibraca limbativentris, the in vitro compatibility between isolated CG 891 of Metarhizium anisopliae and several pesticides used in the culture of irrigated rice was evaluated. The followings parameters were studied: vegetative growth, sporulation and the germination viability in pre- and postcontact of the fungus for two concentrations of fipronil, thiametoxan, fenitrothion, carbofuran, pyrazosulfuron-ethyl, bentazon, clomazone, glyfosate and quinclorac and one concentration of tricyclazole and azoxystrobin. Significant reductions in the vegetative growth and sporulation of $\boldsymbol{M}$. anisopliae were observed for fenitrothion, carbofuran and azoxystrobin. The germination in pre-contact was affected by azoxystrobin. The percentage of germination changed the classification of pesticides in relation to its selectivity on the fungus. However, the results show that in vitro, the pesticides fenitrothion, carbofuran, glyfosate and azoxystrobin are harmful to isolated CG 891 of M. anisopliae.

Keys words: entomopathogens, irrigated rice, integrated pest management, rice stem bug, toxicity.

\section{INTRODUÇÃO}

O inseto-praga T. limbativentris, conhecido como percevejo-do-colmo-do-arroz, tem sua distribuição em todo território nacional, apresentandose como importante praga na cultura do arroz irrigado e causando danos em todo ciclo da cultura (PRANDO et al., 1993). A ocorrência dessa praga tem aumentado expressivamente nas lavouras de arroz irrigado, onde a alta densidade de plantas, o clima e o posicionamento das lavouras favorecem a ocorrência e o aumento populacional do inseto (MARTINS et al., 2004).

Considerando que no manejo fitossanitário das lavouras de arroz, atualmente, preconiza-se a

IDepartamento de Entomologia e Acarologia, Escola Superior de Agricultura “Luiz de Queiroz” (ESALQ), Universidade de São Paulo (USP), 13418-900, Piracicaba, SP, Brasil. E-mail: ftrampelotti@hotmail.com. Autor para correspondência

"Departamento de Genética, ESALQ, USP, Piracicaba, SP, Brasil.

IIIEpagri - Estação Experimental de Itajaí, Itajaí, SC, Brasil.

IVDepartamento de Fitossanidade, Universidade Federal de Pelotas (UFPel), Pelotas, RS, Brasil.

vEmbrapa Clima Temperado - Estação Experimental Terras Baixas, Pelotas, RS, Brasil. 
utilização de agrotóxicos, a avaliação da seletividade a agentes de controle biológico precisa ser considerada, mesmo porque o uso abusivo do controle químico pode acarretar na perda da eficiência sobre o alvo de controle, afetando agentes de controle biológico presentes no ambiente (LOUREIRO et al., 2002). O fungo Metarhizium anisopliae é um dos entomopatógenos incidentes sobre pentatomídeos que atacam o arroz, e a ocorrência natural sobre o percevejo-do-colmo tem sido observada no campo e durante o período de hibernação deste inseto (RAMPELOTTI et al., 2007).

Nesse contexto, este trabalho teve por objetivo avaliar parâmetros biológicos do isolado CG 891 de $\boldsymbol{M}$. anisopliae, in vitro, para determinar sua seletividade a alguns inseticidas, herbicidas e fungicidas utilizados na cultura do arroz irrigado, visando a aprimorar o manejo integrado de $\boldsymbol{T}$. limbativentris.

\section{MATERIAL E MÉTODOS}

Os experimentos foram conduzidos no Laboratório de Produção de Beauveria da Epagri, Itajaí, Santa Catarina (SC). Utilizou-se o isolado CG 891 de $\boldsymbol{M}$. anisopliae (RAMPELOTTI et al., 2007). As colônias utilizadas foram obtidas a partir de repiques do fungo estocado sob refrigeração $\left(10^{\circ} \mathrm{C}\right)$. O meio de cultura utilizado foi batata dextrose ágar (BDA) $\left(\right.$ Merk $\left.^{\circledR}\right)$, e a incubação foi a $27 \pm 1^{\circ} \mathrm{C}$, com 14 horas de fotofase por 12 dias. Os agrotóxicos (tratamentos) testados foram duas doses de: fipronil, tiametoxam, fenitrotiona, carbofurano, pirazossulfurom-etílico, bentazona, clomazona, glifosato, quincloraque; e uma dose de tricyclazole e azoxistrobina (Tabela 1). Os fungicidas triciclazole e azoxistrobina foram escolhidos de acordo com o controle sobre o fitopatógeno Pyricularia grisea (agente causal da brusone do arroz).

Crescimento vegetativo e esporulação: as doses de cada tratamento foram misturadas em meio BDA fundido, sendo vertidos $20 \mathrm{ml}$ em cada placa (Tabela 1). O fungo foi inoculado com agulha de platina, em três pontos equidistantes por placa, totalizando quatro placas por tratamento. Foram obtidas 12 colônias e, após seleção aleatória, oito foram utilizadas para avaliar o crescimento vegetativo, por meio de duas medições transversais (cm). Seis dessas colônias foram selecionadas para determinar a esporulação. Para tanto, recortaram-se as colônias com bisturi, transferindo-as para tubos contendo $10 \mathrm{ml}$ de solução estéril de Tween $80(0,1 \%)$ e agitando-as para facilitar a retirada dos conídios da superfície do meio. A contagem de conídios foi realizada em microscópio óptico na câmara de Neubauer (400X).

Tabela 1 - Agrotóxicos registrados para cultura do arroz irrigado e utilizados nos bioensaios de seletividade.

\begin{tabular}{|c|c|c|c|c|}
\hline & Tratamento & Nome comercial & Categoria $^{3}$ & Doses $\left(\mathrm{ha}^{-1}\right)$ \\
\hline 1 & Fipronil & Standak $250 \mathrm{SC}^{1}$ & I & $160 \mathrm{ml}$ \\
\hline 2 & Fipronil & & I & $240 \mathrm{ml}$ \\
\hline 3 & Tiametoxam & Actara $250 \mathrm{WG}^{1}$ & I & $80 \mathrm{~g}$ \\
\hline 4 & Tiametoxam & & I & $100 \mathrm{~g}$ \\
\hline 5 & Fenitrotiona & Sumithion $500 \mathrm{CE}^{1}$ & I & $1 \mathrm{~L}$ \\
\hline 6 & Fenitrotiona & & I & $1,5 \mathrm{Ll}$ \\
\hline 7 & Pirazossulfuron-etílico & Sirius $250 \mathrm{SC}^{1}$ & I & $60 \mathrm{ml}$ \\
\hline 8 & Pirazossulfuron-etílico & & I & $100 \mathrm{ml}$ \\
\hline 9 & Bentazona & Basagran $600 \mathrm{SL}^{1}$ & $\mathrm{H}$ & $1,2 \mathrm{~L}$ \\
\hline 10 & Bentazona & & $\mathrm{H}$ & $2,0 \mathrm{~L}$ \\
\hline 11 & Carbofurano & Furadan 50 GR $^{2}$ & $\mathrm{H}$ & $5 \mathrm{~kg}$ \\
\hline 12 & Carbofurano & & $\mathrm{H}$ & $8 \mathrm{~kg}$ \\
\hline 13 & Clomazone & Gamit $500 \mathrm{CS}^{2}$ & $\mathrm{H}$ & $0,8 \mathrm{~L}$ \\
\hline 14 & Clomazone & & $\mathrm{H}$ & $1,4 \mathrm{~L}$ \\
\hline 15 & Glifosato & Roundup $360 \mathrm{CS}^{2}$ & $\mathrm{H}$ & $2 \mathrm{~L}$ \\
\hline 16 & Glifosato & & $\mathrm{H}$ & $4 \mathrm{~L}$ \\
\hline 17 & Quincloraque & Facet $500 \mathrm{PM}^{2}$ & $\mathrm{H}$ & $500 \mathrm{~g}$ \\
\hline 18 & Quincloraque & & $\mathrm{H}$ & $750 \mathrm{~g}$ \\
\hline 19 & Triciclazole $^{1}$ & $\operatorname{Bim} 750 \mathrm{BR}(\mathrm{PM})^{2}$ & $\mathrm{~F}$ & $300 \mathrm{~g}$ \\
\hline 20 & Azoxistrobina $^{1}$ & Priori 250 SC $^{2}$ & $\mathrm{~F}$ & $400 \mathrm{~g}$ \\
\hline
\end{tabular}

1 Agrotóxico testado no experimento 1. ${ }^{2}$ Agrotóxico testado no experimento 2. Formulações: SC ou CS=suspensão concentrada, $\mathrm{GR}=$ granulado, $\mathrm{CE}=$ concentrado emulsionável, WG=granulado dispersível, SL=concentrado solúvel, PM=pó molhável. ${ }^{3} \mathrm{I}=$ inseticida; $\mathrm{H}=$ herbicida; $\mathrm{F}=$ fungicida.

Ciência Rural, v.40, n.4, abr, 2010. 
$\mathrm{O}$ valor de $\mathrm{T}$, cuja fórmula é $\mathrm{T}=$ $(20[C V]+80[E S P]) / 100$, foi utilizado para determinar o índice de toxicidade de agrotóxicos sobre o microrganismo (ALVES et al., 1998). O índice biológico de toxicidade proposto por ROSSI-ZALAF et al. (2008) foi utilizado, visando comparar os valores de toxicidade com e sem a inclusão da germinação dos conídios no cálculo. A fórmula do índice biológico proposto por ROSSI-ZALAF et al. (2008) é: IB = $47[\mathrm{CV}]+43[\mathrm{ESP}]+10[\mathrm{GERM}] / 100$, em que IB= índice biológico, $\mathrm{CV}=$ porcentagem de crescimento vegetativo da colônia em relação à testemunha, ESP=porcentagem de esporulação das colônias e GERM= porcentagem de germinação dos conídios em 24 horas. De acordo com a fórmula, o produto pode ser classificado como: tóxico (IB entre 0 e 41 ), moderadamente tóxico (IB entre 42 e 66) e compatível (IB acima de 66).

Germinação dos conídios: a avaliação foi realizada em duas condições: 1) Pré-contato: conídios obtidos de crescimento em meio BDA puro e submetidos ao contato com os agrotóxicos (Tabela 1). Para tanto, preparou-se uma suspensão de $10^{7}$ conídios $\mathrm{ml}^{-1} \mathrm{em}$ água estéril com Tween $80(0,1 \%)$ contendo o agrotóxico testado em sua respectiva dose. Essa suspensão foi mantida sob agitação por uma hora, na temperatura ambiente. Utilizaram-se $100 \mu \mathrm{l}$ para inocular em meio BDA, com auxílio da alça de vidro (Drigalsky). 2) Pós-contato: conídios obtidos do crescimento em BDA com os agrotóxicos. Os conídios foram coletados por raspagem, com auxílio da alça de platina, e transferidos para um tubo de ensaio contendo cinco $\mathrm{ml}$ de uma solução de Tween 80 (0,1\%). Dessa suspensão, $100 \mu$ foram plaqueados em BDA. As condições de incubação foram as mesmas adotadas no ensaio anterior. Tanto na germinação em pré, como em pós-contato, após o período de incubação, determinou-se a germinação dos conídios segundo a metodologia descrita por NEVES et al. (2001).

Análises estatísticas: para todos os parâmetros avaliados, foram instalados dois experimentos em delineamento experimental inteiramente casualizado, com seis e sete tratamentos, respectivamente, com quatro repetições. Os dados obtidos foram transformados pela função $\sqrt{x+0,5}$ e submetidos à análise de variância e ao teste Tukey $(\alpha<0,05)$, sendo utilizado o software estatístico Statistica. A normalidade foi aferida pelo Teste de Shapiro Wilk, e a homocedasticidade, pelo Teste de Bartlett. Para os parâmetros que não apresentaram distribuição normal, foi utilizado o teste de comparação múltipla de Kruskall-Wallis (Software R).

\section{RESULTADOS E DISCUSSÃO}

Efeito sobre crescimento vegetativo e esporulação: para os tratamentos com inseticidas, ambas as doses de fipronil e tiametoxam não reduziram o crescimento vegetativo de $\boldsymbol{M}$. anisopliae isolado CG 891 (Tabela 2). Essa redução foi observada para carbofurano (5 e $8 \mathrm{~kg} \mathrm{ha}^{-1}$ ) e para fenitrotiona, na dose $1,5 \mathrm{~L} \mathrm{ha}^{-1}$. Em relação a conidiogênese, carbofurano $5 \mathrm{e}$ $8 \mathrm{~kg} \mathrm{ha}^{-1}$ reduziram significativamente a esporulação de M. anisopliae em comparação com o grupo controle. Para os demais inseticidas, exceto fenitrotiona na dose de $1,5 \mathrm{~L} \mathrm{ha}{ }^{-1}$, não se observou redução do número de conídios produzidos. BATISTA FILHO et al. (2001), utilizando a mesma formulação adotada neste trabalho, relataram que tiametoxam não reduz o número de conídios e o diâmetro das colônias de $\boldsymbol{M}$. anisopliae e de outros entomopatógenos. Assim, considerando-se a diversidade do isolado testado, pode-se inferir que esse inseticida apresenta-se compatível com a espécie M. anisopliae, nas condições testadas. Nesse mesmo trabalho, o efeito de fipronil sobre a redução da esporulação, o crescimento e o número de unidades formadoras de colônias de $\boldsymbol{M}$. anisopliae foi observado para uma formulação em grânulos dispersíveis em água com concentração de $800 \mathrm{~g} \mathrm{~kg}^{-1}$ de produto, mostrando que a seletividade dos microrganismos é dependente da concentração de ingrediente ativo e da formulação utilizada, provavelmente pela presença de componentes inertes presentes nessas formulações que podem atuar de forma a maximizar ou minimizar o efeito do produto sobre o microrganismo.

Pirazossulfurom-etílico, na dose $100 \mathrm{ml} \mathrm{ha}^{-1}$, causou uma redução acentuada no crescimento vegetativo do fungo (Tabela 2). O contrário pode ser observado para os demais herbicidas e doses testadas. Com relação à esporulação, pode-se constatar que não existe uma relação direta entre o tamanho da colônia e a quantidade de conídios produzidos, pois, para o herbicida pirazossulfurom-etílico, não foi observada diferença do número de conídios quando comparados ao controle. Na dose 1,4L ha- ${ }^{-1}$ de clomazone e em ambas as doses de glifosato ( 2 e $4 \mathrm{~L} \mathrm{ha}^{-1}$ ) e quincloraque (500 e $\left.750 \mathrm{~g} \mathrm{ha}^{-1}\right)$, reduções foram observadas na esporulação.

O efeito dos fungicidas testados sobre o isolado CG 891 de $\boldsymbol{M}$. anisopliae demonstrou o esperado em relação ao grupo químico utilizado. Observou-se que triciclazole não afeta e azoxistrobina reduz significativamente o crescimento vegetativo. $\mathrm{Na}$ esporulação, observou-se uma redução acentuada para ambos os fungicidas, sendo azoxistrobina mais tóxico (Tabela 2). 
Tabela 2 - Crescimento médio das colônias (cm) e número médio de conídios $\mathrm{ml}^{-1} \pm$ EP do isolado CG 891 de Metarhizium anisopliae crescido em meio contendo agrotóxicos.

\begin{tabular}{|c|c|c|c|c|c|}
\hline \multirow{2}{*}{$\begin{array}{c}\text { Tratamento } \\
----------- \\
\text { Controle }\end{array}$} & \multirow{2}{*}{$\begin{array}{c}\text { Doses }\left(\mathrm{ha}^{-1}\right) \\
-\end{array}$} & \multicolumn{2}{|c|}{ Diâmetro (cm) } & \multicolumn{2}{|c|}{ Conídios $\left(10^{7}\right.$ conídios $\left.\mathrm{ml}^{-1}\right)$} \\
\hline & & $4,55 \pm 0,05$ & $\mathrm{ab}$ & $8,51 \pm 0,19$ & a \\
\hline \multirow[t]{2}{*}{ Fipronil } & $160 \mathrm{ml}$ & $4,54 \pm 0,06$ & $\mathrm{ab}$ & $6,53 \pm 1,14$ & $\mathrm{ab}$ \\
\hline & $240 \mathrm{ml}$ & $4,61 \pm 0,08$ & $\mathrm{ab}$ & $7,05 \pm 0,14$ & $\mathrm{ab}$ \\
\hline \multirow[t]{2}{*}{ Tiametoxam } & $80 \mathrm{~g}$ & $4,62 \pm 0,03$ & $\mathrm{ab}$ & $7,13 \pm 0,97$ & $\mathrm{ab}$ \\
\hline & $100 \mathrm{~g}$ & $4,52 \pm 0,06$ & ab & $6,63 \pm 0,46$ & ab \\
\hline \multirow[t]{2}{*}{ Fenitrotiona } & $1 \mathrm{~L}$ & $4,14 \pm 0,06$ & bc & $4,70 \pm 0,27$ & ab \\
\hline & $1,5 \mathrm{~L}$ & $3,97 \pm 0,11$ & c & $3,79 \pm 0,71$ & $\mathrm{~b}$ \\
\hline \multirow[t]{2}{*}{ Pirazossulfuron-etílico } & $60 \mathrm{ml}$ & $4,68 \pm 0,07$ & a & $7,03 \pm 0,40$ & $\mathrm{ab}$ \\
\hline & $100 \mathrm{ml}$ & $3,81 \pm 0,23$ & d & $5,29 \pm 1,68$ & $a b$ \\
\hline \multirow[t]{2}{*}{ Bentazona } & $1,2 \mathrm{~L}$ & $4,40 \pm 0,11$ & abc & $6,85 \pm 0,73$ & $\mathrm{ab}$ \\
\hline & $2,0 \mathrm{~L}$ & $4,50 \pm 0,08$ & $\mathrm{ab}$ & $7,84 \pm 0,50$ & a \\
\hline CV (\%) & \multicolumn{3}{|c|}{3,09} & \multicolumn{2}{|c|}{10,91} \\
\hline Controle & - & $4,89 \pm 0,03$ & a & $15,50 \pm 2,00$ & a \\
\hline \multirow[t]{2}{*}{ Carbofurano } & $5 \mathrm{~kg}$ & $4,26 \pm 0,08$ & c & $5,04 \pm 0,15$ & e \\
\hline & $8 \mathrm{~kg}$ & $4,41 \pm 0,12$ & bc & $5,54 \pm 0,70$ & de \\
\hline \multirow[t]{2}{*}{ Clomazone } & $0,8 \mathrm{~L}$ & $4,79 \pm 0,08$ & $\mathrm{a}$ & $11,52 \pm 1,23$ & $\mathrm{ab}$ \\
\hline & $1,4 \mathrm{~L}$ & $4,78 \pm 0,06$ & a & $10,12 \pm 1,30$ & bc \\
\hline \multirow[t]{2}{*}{ Glifosato } & $2 \mathrm{~L}$ & $4,78 \pm 0,05$ & a & $7,15 \pm 0,63$ & cde \\
\hline & $4 \mathrm{~L}$ & $4,57 \pm 0,08$ & $a b c$ & $8,96 \pm 0,59$ & bcd \\
\hline \multirow[t]{2}{*}{ Quincloraque } & $500 \mathrm{~g}$ & $4,74 \pm 0,06$ & $a b c$ & $9,85 \pm 0,90$ & bc \\
\hline & $750 \mathrm{~g}$ & $4,72 \pm 0,09$ & abc & $9,15 \pm 0,37$ & bcd \\
\hline Triciclazole $^{1}$ & $300 \mathrm{~g}$ & $4,77 \pm 0,04$ & $\mathrm{a}$ & $9,11 \pm 0,53$ & bcd \\
\hline Azoxistrobina $^{1}$ & $400 \mathrm{~g}$ & $3,91 \pm 0,07$ & d & $5,61 \pm 0,42$ & de \\
\hline CV (\%) & & 2,03 & & 8,3 & \\
\hline
\end{tabular}

Médias \pm EP seguidas da mesma letra nas colunas não diferem entre si pelo teste de Tukey, a 5\% de significância. Médias transformadas pela $\sqrt{x+0,5}$. ${ }^{1}$ dose única.

Com base no valor de T, constatou-se que os inseticidas fenitrotiona e carbofurano nas doses máximas e glifosato na dose mínima foram moderadamente tóxicos e que carbofurano na dose mínima e azoxistrobina foram incompatíveis com o isolado CG 891 de M. anisopliae. Resultados encontrados em outros trabalhos demonstraram que a formulação utilizada pode determinar o nível de toxicidade do agrotóxico, o que seria explicado primeiramente pela quantidade de ingrediente ativo da fórmula e pelos ingredientes inertes presentes nesta, e também, pela espécie e/ou pelo isolado do entomopatógeno. A compatibilidade de carbofurano, numa formulação em suspensão concentrada (350g ingrediente ativo), e glifosato foram observados para Beauveria bassiana (ANDALÓ et al., 2004).

Para ambas as doses de carbofurano (incompatível e moderadamente tóxica ao isolado testado), pode-se observar que a dose mínima foi mais tóxica que a dose máxima. No entanto, na análise do valor de $\mathrm{T}$ e dos critérios de classificação para esse inseticida, pode-se considerar que esses valores foram próximos, não diferindo estatisticamente nas análises para o crescimento vegetativo e a esporulação. Para glifosato (moderadamente tóxico na dose mínima e compatível na dose máxima), esse resultado também foi observado.

No entanto, a utilização do índice biológico (IB) mostrou que nenhum dos agrotóxicos testados foi tóxico ao isolado CG 891, ou seja, carbofurano nas duas doses e azoxistrobina que se apresentaram como incompatíveis para a classificação anterior (ALVES et al., 1998) apresentaram-se como moderadamente tóxicos quando se utilizou o IB (Tabela 3). Na análise dos valores de germinação em pré-contato, pode-se constatar que o inseticida carbofurano não afetou a germinação, assim, ao incluir a germinação dos conídios na fórmula, obteve-se o aumento do valor do IB, alterando sua classificação. Glifosato e fenitrotiona passaram de moderadamente tóxicos para compatíveis. 
Tabela 3 - Valores de T e classificação da toxicidade entre alguns agrotóxicos utilizados na cultura do arroz irrigado e o isolado CG 891 de Metarhizium anisopliae.

\begin{tabular}{|c|c|c|c|c|c|}
\hline Tratamento & Doses $\left(h^{-1}\right)$ & $\mathrm{T}^{1}$ & Classificação $^{1}$ & $\mathrm{~T}^{2}$ & Classificação $^{2}$ \\
\hline \multirow[t]{2}{*}{ Fipronil } & $160 \mathrm{ml}$ & 81,33 & $\mathrm{C}$ & 87,49 & $\mathrm{C}$ \\
\hline & $240 \mathrm{ml}$ & 86,45 & $\mathrm{C}$ & 89,94 & $\mathrm{C}$ \\
\hline \multirow[t]{2}{*}{ Tiametoxam } & $80 \mathrm{~g}$ & 87,34 & $\mathrm{C}$ & 91,45 & $\mathrm{C}$ \\
\hline & $100 \mathrm{~g}$ & 82,19 & $\mathrm{C}$ & 87,19 & $\mathrm{C}$ \\
\hline \multirow[t]{2}{*}{ Fenitrotiona } & $1 \mathrm{~L}$ & 62,26 & $\mathrm{C}$ & 72,51 & $\mathrm{C}$ \\
\hline & $1,5 \mathrm{~L}$ & 53,04 & MT & 66,66 & $\mathrm{C}$ \\
\hline \multirow[t]{2}{*}{ Carbofurano } & $5 \mathrm{~kg}$ & 43,45 & I & 63,53 & MT \\
\hline & $8 \mathrm{~kg}$ & 46,60 & MT & 65,26 & MT \\
\hline \multirow[t]{2}{*}{ Pirazossulfuron-etílico } & $60 \mathrm{ml}$ & 87 & $\mathrm{C}$ & 90,36 & $\mathrm{C}$ \\
\hline & $100 \mathrm{ml}$ & 66 & $\mathrm{C}$ & 71,59 & $\mathrm{C}$ \\
\hline \multirow[t]{2}{*}{ Bentazona } & $1,2 \mathrm{~L}$ & 83,71 & $\mathrm{C}$ & 87,56 & $\mathrm{C}$ \\
\hline & $2,0 \mathrm{~L}$ & 93,46 & $\mathrm{C}$ & 93,50 & $\mathrm{C}$ \\
\hline \multirow[t]{2}{*}{ Clomazone } & $0,8 \mathrm{~L}$ & 79,04 & $\mathrm{C}$ & 85,00 & $\mathrm{C}$ \\
\hline & $1,4 \mathrm{~L}$ & 71,77 & $\mathrm{C}$ & 80,02 & $\mathrm{C}$ \\
\hline \multirow[t]{2}{*}{ Glifosato } & $2 \mathrm{~L}$ & 56,42 & MT & 72,38 & $\mathrm{C}$ \\
\hline & $4 \mathrm{~L}$ & 64,89 & $\mathrm{C}$ & 75,38 & $\mathrm{C}$ \\
\hline \multirow[t]{2}{*}{ Quincloraque } & $500 \mathrm{~g}$ & 70,21 & $\mathrm{C}$ & 77,28 & $\mathrm{C}$ \\
\hline & $750 \mathrm{~g}$ & 66,51 & $\mathrm{C}$ & 74,25 & $\mathrm{C}$ \\
\hline Triciclazole & $300 \mathrm{~g}$ & 66,51 & $\mathrm{C}$ & 77,02 & $\mathrm{C}$ \\
\hline Azoxistrobina & $400 \mathrm{~g}$ & 44,92 & I & 57,34 & MT \\
\hline
\end{tabular}

${ }^{1}$ Valores de T e classificação do produto: 0-45 incompatível (I); 46-60 moderadamente tóxico (MT); >60 compatível (C), determinados segundo ALVES et al. (1998). ${ }^{2}$ Valores de T e classificação do produto: 0-45 incompatível (I); 46-60 moderadamente tóxico (MT); >60 compatível (C), determinados segundo ROSSI-ZALAF et al. (2008).

Ambos os agrotóxicos não afetaram a germinação dos conídios, mas o valor do IB aumentou em razão da alteração do valor porcentual de cada parâmetro na fórmula. A influência do impacto de agrotóxicos na germinação dos conídios altera a classificação toxicológica dos agrotóxicos com relação à seletividade.

Os demais agrotóxicos e suas respectivas doses testadas, foram compatíveis com o fungo (Tabela 3). Esses resultados corroboram com o que tem sido comum em trabalhos semelhantes que utilizam a mesma metodologia de avaliação do efeito de produtos fitossanitários sobre os entomopatógenos in vitro (LOUREIRO et al., 2002).

Efeito sobre a germinação dos conídios: para germinação em pré-contato, apenas o fungicida azoxistrobina reduziu a germinação dos conídios. Para os demais agrotóxicos, não houve reduções significativas, independentemente da dose testada sobre o fungo (Tabela 4). Apesar da compatibilidade da maioria dos agrotóxicos e suas respectivas doses testadas ao isolado CG 891, em outros trabalhos podese observar que alguns inseticidas na mesma formulação usada neste trabalho foram tóxicos a outros isolados de M. anisopliae (BATISTA FILHO et al., 2001).
Nos tratamentos com inseticidas em póscontato, tiametoxam em ambas as doses testadas e carbofurano na dose máxima, foi observada redução na germinação do fungo com relação ao observado na germinação em pré-contato. Já para fipronil e fenitrotiona a viabilidade não foi alterada (Tabela 4).

A permanência de redução na germinação foi observada para pirazossulfurom-etílico na dose máxima e bentazona, clomazone e glifosato em ambas as doses. Quincloraque não afetou a capacidade germinativa dos conídios na menor dose.

Ambos os fungicidas mostraram redução da germinação para viabilidade em pré-contato. No entanto, observou-se uma recuperação da viabilidade dos conídios na viabilidade em pré-contato (Tabela 4). O contato do fungo com alguns dos agrotóxicos, durante determinado período, permitiu que o microrganismo produzisse conídios viáveis, sugerindo que frente a uma fonte estressora o microrganismo recuperou-se, o que poderia demonstrar a capacidade adaptativa em relação ao meio (AZEVEDO et al., 2002).

Na cultura do arroz irrigado, diferentes formulações e estratégias de manejo são utilizadas. É exemplo desse esforço o uso do inseticida fipronil para o tratamento de sementes e do carbofurano, na 
Tabela 4 - Germinação de conídios de Metarhizium anisopliae isolado CG 891 em pré e pós-contato \pm EP para alguns agrotóxicos utilizados na cultura do arroz irrigado.

\begin{tabular}{|c|c|c|c|}
\hline Tratamento & Doses $\left(\mathrm{ha}^{-1}\right)$ & Germinação pré-contato & Germinação pós-contato \\
\hline \multicolumn{4}{|c|}{ 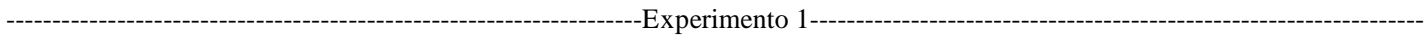 } \\
\hline Controle & - & $91 \pm 0,06(439)^{2} \quad \mathrm{ab}$ & $85 \pm 2,29 \quad a$ \\
\hline \multirow[t]{2}{*}{ Fipronil } & $160 \mathrm{ml}$ & $76 \pm 0,06(368) \quad a b$ & $73 \pm 2,54$ abcde \\
\hline & $240 \mathrm{ml}$ & $67 \pm 0,10(414) \quad a b$ & $78 \pm 2,75$ abc \\
\hline \multirow[t]{2}{*}{ Tiametoxam } & $80 \mathrm{~g}$ & $77 \pm 0,03(505) \quad b$ & $66 \pm 1,75$ cdef \\
\hline & $100 \mathrm{~g}$ & $70 \pm 0,19(418,5) \quad a b$ & $62 \pm 2,36$ ef \\
\hline \multirow[t]{2}{*}{ Fenitrotiona } & $1 \mathrm{~L}$ & $60 \pm 0,06(162,5) \quad a b$ & $83 \pm 1,28 \quad a b$ \\
\hline & $1,5 \mathrm{~L}$ & $65 \pm 0,12(132) \quad$ a & $77 \pm 3,25$ abcde \\
\hline \multirow[t]{2}{*}{ Pirazossulfurom-etílico } & $60 \mathrm{ml}$ & $65 \pm 0,28(523,5) \quad b$ & $92 \pm 1,91 \quad a b$ \\
\hline & $100 \mathrm{ml}$ & $55 \pm 0,32(96,5) \quad$ a & $61 \pm 1,66 \mathrm{f}$ \\
\hline \multirow[t]{2}{*}{ Bentazona } & $1,2 \mathrm{~L}$ & $75 \pm 0,13(343,5) \quad a b$ & $66 \pm 2,36$ def \\
\hline & $2,0 \mathrm{~L}$ & $74 \pm 0,08(379) \quad a b$ & $72 \pm 3,77$ bcdef \\
\hline CV (\%) & & 3,72 & 6,71 \\
\hline Controle & - & $85 \pm 0,14(502) \quad b$ & $92 \pm 3,55 \quad a$ \\
\hline \multirow[t]{2}{*}{ Carbofurano } & $5 \mathrm{~kg}$ & $86 \pm 0,07(140) \quad a b$ & $80 \pm 2,30 \quad a b$ \\
\hline & $8 \mathrm{~kg}$ & $75 \pm 0,07(223,5) \quad a b$ & $68 \pm 5,12$ bc \\
\hline \multirow[t]{2}{*}{ Clomazone } & $0,8 \mathrm{~L}$ & $70 \pm 0,23(462) \quad b$ & $71 \pm 2,30 \quad$ bc \\
\hline & $1,4 \mathrm{~L}$ & $60 \pm 0,19(460) \quad b$ & $60 \pm 3,71 \quad$ с \\
\hline \multirow[t]{2}{*}{ Glifosato } & $2 \mathrm{~L}$ & $66 \pm 0,15(442,5) \quad b$ & $68 \pm 3,74$ bc \\
\hline & $4 \mathrm{~L}$ & $66 \pm 0,05(305,5) \quad a b$ & $67 \pm 4,60 \quad$ cd \\
\hline \multirow[t]{2}{*}{ Quincloraque } & $500 \mathrm{~g}$ & $44 \pm 0,31(408) \quad a b$ & $76 \pm 2,60 \quad a b$ \\
\hline & $750 \mathrm{~g}$ & $35 \pm 0,14(422) \quad a b$ & $70 \pm 2,48$ bc \\
\hline Triciclazole $^{1}$ & $300 \mathrm{~g}$ & $59 \pm 0,25(443) \quad b$ & $73 \pm 2,71 \quad$ bc \\
\hline Azoxistrobina $^{1}$ & $400 \mathrm{~g}$ & $42 \pm 0,11(50) \quad a$ & $72 \pm 1,32$ bc \\
\hline CV (\%) & & 4,40 & 4,62 \\
\hline
\end{tabular}

Percentagens médias \pm EP seguidas da mesma letra nas colunas não diferem entre si pelo teste de comparação múltipla de Kruskall Wallis, a $5 \%$ de significância. ${ }^{1}$ dose única. ${ }^{2}$ valores entre parênteses: soma de ranks.

formulação granulada, no controle da bicheira-da-raiz (Oryzophagus oryzae) (Coleoptera:Curculionidae). Apesar desses inseticidas não serem utilizados em pulverizações, o seu contato com potenciais agentes de controle biológico poderia causar interferência sobre o entomopatógeno presente no solo, um dos principais locais de permanência de microrganismos. Além da temperatura e umidade, a interferência de agentes abióticos, como os agrotóxicos, reduz a sobrevivência de propágulos no solo ou sobre cadáveres de hospedeiros, prejudicando a relações patógeno/ hospedeiro e a manutenção de epizootias (AZEVEDO et al., 2002).

Portanto, estudos complementares em semicampo e campo do efeito dos agrotóxicos moderadamente tóxicos e incompatíveis utilizados neste trabalho são necessários para se buscar entender a real compatibilidade com o fungo. A implantação de uma estratégia de manejo para o controle do inseto $\boldsymbol{T}$. limbativentris utilizando o isolado CG 891 de $\boldsymbol{M}$. anisopliae requer cuidados especiais quanto à utilização de alguns agrotóxicos. Avaliações realizadas em campo com M. anisopliae e B. bassiana sugerem baixa taxa de infecção dentro das parcelas avaliadas, apesar dos arrozais serem bastante favoráveis à disseminação do entomopatógeno (MARTINS et al., 2004). No entanto, os sítios de hibernação e as taipas caracterizam locais importantes para permanência dos entomopatógenos sobre cadáveres dos insetos durante períodos de entressafra. Nesse período, os percevejos migram para vegetação próxima e permanecem agrupados na base de gramíneas, facilitando assim a contaminação com o entomopatógeno.

A constatação da toxicidade in vitro subsidiará experimentações no campo, onde os efeitos das interações entre o entomopatógeno e os agrotóxicos sobre um alvo serão determinados, levando 
à escolha de agrotóxicos mais seletivos e ao delineamento de uma estratégia para o manejo integrado de insetos-praga na cultura do arroz irrigado.

\section{CONCLUSÕES}

Fenitrotiona, carbofurano, glifosato e azoxistrobina afetam parâmetros biológicos avaliados do isolado CG 891 de $\boldsymbol{M}$. anisopliae. A inserção da germinação no cálculo de toxicidade altera a classificação do agrotóxico com relação a sua seletividade sobre o entomopatógeno.

\section{AGRADECIMENTOS}

Ao Conselho Nacional de Desenvolvimento Científico e Tecnológico do Brasil (CNPq), pela concessão de bolsa de estudos para a primeira autora e ao engenheiro agrônomo MSc. Rafael Major Pitta, pelo auxílio nas análises estatísticas.

\section{REFERÊNCIAS}

ALVES, S.B. et al. Produtos fitossanitários e entomopatógenos. In: ALVES, S.B. (Ed.). Controle microbiano de insetos. Piracicaba: FEALQ, 1998. p.217-238.

ANDALÓ, V. et al. Compatibilidade de Beauveria bassiana com agroquímicos visando o controle da cochonilha-da-raizdo-cafeeiro Dysmicoccus texensis Tinsley (Hemiptera: Pseudococcidae). Neotropical Entomology, Londrina, v.33, p.463-467, 2004. Disponível em: <http://www.scielo.br/ s cielo.ph p ? s crip t = s ci_art text \& pid = S 1519 566X2004000400011\&lng=pt\&nrm=iso $>$. Acesso em: 01 fev. 2010. doi: 10.1590/S1519-566X2004000400011.

AZEVEDO, J.L. de et al. Melhoramento de fungos de importância na agricultura. In: MELO, I.S. de et al. (Eds). Recursos genéticos e melhoramento - microrganismos. Jaguariúna: Embrapa Meio Ambiente, 2002. p.323-355.

BATISTA FILHO et al. Effect of thiamethoxam on entomopathogenic microorganisms. Neotropical Entomology, Londrina, v.30, p.437-447, 2001. Disponível em: <http://
www.scielo.br/scielo.php?script=sci_arttext\&pid=S1519566X2001000300017\&lng=pt\&nrm=iso. Acesso em: $01 \mathrm{fev}$ 2010. doi: 10.1590/S1519-566X2001000300017.

LOUREIRO, E. de S. et al. Efeito de produtos fitossanitários químicos utilizados em alface e crisântemo sobre fungos entomopatogênicos. Neotropical Entomology, Londrina, v.30, p.263-269, 2002. Disponível em: <http://www.scielo.br/ scielo.ph p ? s cript = sci_art text \& pid = S 1519 566X2002000200014\&lng=pt\&nrm=iso $>$. Acesso em: $01 \mathrm{fev}$ 2010. doi: 10.1590/S1519-566X2002000200014.

MARTINS, J.F. da S. et al. Eficiência de Metarhizium anisopliae no controle do percevejo-do-colmo Tibraca limbativentris (Heteroptera: Pentatomidae) em lavoura de arroz irrigado. Ciência Rural, Santa Maria, v.34, p.1681-1688, 2004. Disponível em: <http://www.scielo.br/scielo.php?pid=S010384782004000600003\&script=sci_arttext $>$. Acesso em: $01 \mathrm{fev}$ 2010. doi: 10.1590/S0103-84782004000600003.

NEVES, P.M.O.J. et al. Compatibility of entomopathogenic fungi with neonicotinoid insecticides. Neotropical Entomology, Londrina, v.30, p.263-268, 2001. Disponiível em: <http:// www.scielo.br/scielo.php?script $=$ sci_arttext \&pid $=$ S1519566X2001000200009\&lng $=p t \& n r m=i s o>$. Acesso em: $01 \mathrm{fev}$ 2010. doi: 10.1590/S1519-566X2001000200009.

PRANDO, H.F. et al. Ciclo de vida de Tibraca limbativentris Stal, 1860 (Hemiptera: Pentatomidae) em condições de laboratório. Revista Brasileira de Entomologia, São Paulo, v.37, p.335-339, 1993.

R DEVELOPMENT CORE TEAM. R: a language and environment for statistical computing. Vienna, Austria: Foundation for Statistical Computing, 2008. ISBN 3-90005107-0, http://www.R-project.org.

RAMPELOTTI, F.T. et al. Patogenicidade de Metarhizium anisophiae (Metsch.) Sorokin sobre as fases do desenvolvimento de Tibraca limbativentris Stal (Hemiptera: Pentatomidae) em condições de laboratório. Arquivos do Instituto Biológico. São Paulo, v.74, p.141-148, 2007. Disponível em: <http:// www.biologico.sp.gov.br/rev_arq.php?vol=74\&num=2>. Acesso em: 01 fev. 2010.

ROSSI-ZALAF, L.S. et al. Interação de microrganismos com outros agentes de controle de pragas e doenças. In: ALVES, S.B.; LOPES, R.B. (Eds.). Controle microbiano de pragas na América Latina: Avanços e Desafios. Piracicaba: FEALQ, 2008. p.279-302. 